\title{
WiP Abstract: On the Need for Sensor and Actuator Placement Algorithms in Nonlinear Systems
}

\author{
Sebastian A. Nugroho \\ sebastian.nugroho@my.utsa.edu \\ The University of Texas at San Antonio \\ San Antonio, Texas
}

\author{
Ahmad F. Taha \\ ahmad.taha@utsa.edu \\ The University of Texas at San Antonio \\ San Antonio, Texas
}

\begin{abstract}
In this work, we consider the problem of selecting-or placingsensors and control nodes in nonlinear dynamic networks (NDN) which model the evolution of many cyber-physical systems. Specifically, we illustrate here the need for computational methods that obtain the optimal combinations of sensors and actuators, while considering the nonlinear behavior of NDN and and systems-theoretic performance metrics. This work-in-progress abstract briefly showcases our recent work in this problem, which is divided into two steps: (1) Characterizing the classes of nonlinearities encompassed in NDN; (2) Developing tractable computational algorithms to solve the joint problem of selecting control nodes/sensors and designing feedback control/state estimators.
\end{abstract}

\section{CCS CONCEPTS}

- Mathematics of computing $\rightarrow$ Mixed discrete-continuous optimization.

\section{KEYWORDS}

Control nodes selection, sensors selection, nonlinear dynamic networks, cyber-physical systems control and estimation.

\section{ACM Reference Format:}

Sebastian A. Nugroho and Ahmad F. Taha. 2019. WiP Abstract: On the Need for Sensor and Actuator Placement Algorithms in Nonlinear Systems. In ICCPS '19: ACM/IEEE International Conference on Cyber-Physical Systems, April 16-18, 2019, Montreal, QC, Canada. ACM, New York, NY, USA, 2 pages. https://doi.org/10.1145/3302509.3313313

\section{INTRODUCTION AND MOTIVATION}

Intelligent transportation systems, water networks, power grids, and many other cyber-physical systems (CPS) are naturally assembled into nonlinear dynamic networks (NDN)-intertwined networks that evolve nonlinearly in time and space. A defining feature of these networks is the prevalence and ubiquity of sensors and control nodes/actuators: sensors collecting data while ensuring network observability, and controllers (or actuators) driving networks to desired states. The selection/placement of sensors and control

This material is based upon work supported by the National Science Foundation under Grant CMMI-DCSD-1728629.

Permission to make digital or hard copies of part or all of this work for personal or classroom use is granted without fee provided that copies are not made or distributed for profit or commercial advantage and that copies bear this notice and the full citation on the first page. Copyrights for third-party components of this work must be honored

For all other uses, contact the owner/author(s).

ICCPS '19, April 16-18, 2019, Montreal, QC, Canada

(c) 2019 Copyright held by the owner/author(s).

ACM ISBN 978-1-4503-6285-6/19/04.

https://doi.org/10.1145/3302509.3313313

Table 1: SCSP in various CPS.

\begin{tabular}{|c|c|c|}
\hline Application & Actuator Selection & Sensor Selection \\
\hline $\begin{array}{l}\text { Transportation Net- } \\
\text { works }[1,3]\end{array}$ & $\begin{array}{l}\text { Closure/opening of } \\
\text { roads, traffic lights }\end{array}$ & $\begin{array}{l}\text { Placement of sensors } \\
\text { monitoring traffic } \\
\text { flow }\end{array}$ \\
\hline Power Grids [7] & $\begin{array}{l}\text { Selection of genera- } \\
\text { tors, batteries }\end{array}$ & $\begin{array}{l}\text { Placement of phasor } \\
\text { measurement units }\end{array}$ \\
\hline
\end{tabular}

nodes (SC) is one of the main steps in the network design and control. From this perspective, the SC selection problems (SCSP) can be split into: (i) finding SC selection/placement satisfying system constraints and performance metrics, while (ii) designing localized control/estimation routines. Tab. 1 describes two examples of SCSP in CPS.

To illustrate the importance of studying SCSP for say, any nonlinear dynamical systems, suppose that we want to perform state estimation while obtaining the minimal number of sensor on a permanent magnet stepper motor [8] which dynamics are expressed as $\dot{\mathbf{x}}(t)=\phi(\mathbf{x})+\mathbf{B u}(t), \mathbf{y}(t)=\mathbf{C x}(t)$, where $\phi: \mathbb{R}^{4} \rightarrow \mathbb{R}^{4}$ represents the nonlinear dynamics specified as

$$
\phi(\mathbf{x})=\left[\begin{array}{c}
x_{2}(t) \\
-K_{1} x_{2}(t)-K_{2} x_{3} \sin \left(K_{3} x_{1}(t)\right)+K_{2} x_{4} \cos \left(K_{3} x_{1}(t)\right) \\
K_{4} x_{2}(t) \sin \left(K_{3} x_{1}(t)\right)-K_{5} x_{3}(t) \\
-K_{4} x_{2}(t) \cos \left(K_{3} x_{1}(t)\right)-K_{5} x_{4}(t)
\end{array}\right] .
$$

The simplest approach to solve this problem is to linearize the above NDN around known equilibrium points, in which methods to solve

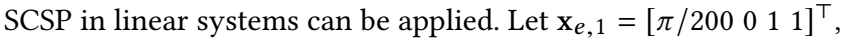
and $\mathbf{x}_{e, 2}=\left[\begin{array}{llll}-\pi & 0 & 0 & 0\end{array}\right]^{\top}$ be two equilibria of the desired operating regions. After linearizing the system around these points, we obtain linear dynamics $\dot{\mathbf{x}}(t)=\mathbf{A}_{e, i} \mathbf{x}(t)+\mathbf{B u}(t)$, for $i=1,2$. The observer design along with sensors selection can be lumped into the following nonconvex feasibility problem

$$
\mathbf{A}_{e, i}^{\top} \mathbf{P}+\mathbf{P A}_{e, i}-\mathbf{C}^{\top} \Gamma \mathbf{Y}^{\top}-\mathrm{Y} \Gamma \mathrm{C} \prec 0, \quad \mathbf{P}>0,
$$

where $\mathbf{P}, \mathrm{Y}$, and $\Gamma$ are matrix variables to be solved. Specifically, $\Gamma=\operatorname{diag}\left(\left[\gamma_{1}, \ldots, \gamma_{4}\right]\right)$ is a diagonal matrix representing the binary sensors selection, with $\gamma_{i}=1$ if sensor $i$ is selected and zero otherwise. After solving the above mixed-integer semi-definite program (MI-SDP) using branch-and-bound (BnB) algorithm, we obtain two distinct locations of sensor for the two linear models: $\gamma_{(1)}^{*}=\{0,1,0,0\}, \gamma_{(2)}^{*}=\{1,0,0,0\}$. It is revealed that the second sensor selection, $\gamma_{(2)}^{*}$, can be used to design observers for linear systems linearized around both equilibria, but not otherwise. Interestingly, the sensor selection for the original nonlinear system using Lipschitz-based observer-see Section 2.2-is identical to $\gamma_{(2)}^{*}$. This simple example empirically illustrates that the solution to a SCSP depends on the local behavior of the network. In contrast, the 
biggest advantage of solving SCSP for NDN is the independence of the sensor/actuator selection from the NDN's operating point, which consequently offers practicality in situations where operating points of NDN are frequently changing. It is also important to mention that for NDN comprising of hundreds/thousands of subsystems, SCSP is highly non trivial, particularly when a specific metric such as robustness is considered.

\section{RESEARCH METHODOLOGY}

The optimal selection/placement of SC in linear networks has been thoroughly studied in the literature through various approaches $[6,7]$. Despite these advances, the SCSP for NDN remains to a large extent an open research problem. To the best of our knowledge, there are only two main approaches in the literature that are devoted to solve SCSP for NDN. The first approach is based on empirical Gramians in low-dimensional systems [5], while the other presents a method for reconstructing the initial states $\mathbf{x}\left(t_{0}\right)$ of NDN, given a NDN model, and optimally selecting sensors given an observation window of fixed length [2]. Still, these approaches are limited in their applicability to large-scale, unstable networks, and difficulty to incorporate SC selection or robustness metrics. To that end, the objective of this work is to develop control-theoretic based methods for some classes of SCSP in various NDN while addressing the limitations of the aforementioned approaches. The following sections give a more detailed description of our approaches.

\subsection{Classification of The Nonlinear Dynamics}

We start this section by exploiting the following attributes of NDN: (i) physical states are almost always bounded, and (ii) the nonlinearities present in the NDN can be shown to belong to various classes of nonlinear functions. For instance, NDN with continuous partial derivatives on compact domains are indeed locally Lipschitz continuous. Knowing that a NDN satisfies the Lipschitz property is crucial as it allow us to design state estimator/controller for Lipschitz nonlinear systems, for which strategies for solving SCSP can be applied-see Section 2.2. However, this method often relies on the knowledge of the corresponding Lipschitz constant. Although computing Lipschitz constant analytically for NDN of medium size and complexity can still be manageable (see our recent work on analytical computation of Lipschitz constants [3, 4]), for highdimensional NDN with complex nonlinearities (e.g. multi-machine power networks), computing Lipschitz constants can be daunting. This observation motivates us to develop a new strategy to determine Lipschitz constants for high-dimensional NDN. To begin with, let $f: \mathbb{R}^{n} \rightarrow \mathbb{R}$ be a differentiable function with domain of interest $\mathcal{X} \subset \mathbb{R}^{n}$. Since $f$ is locally Lipschitz in $\mathcal{X}$, then we have

$$
\|f(\mathbf{x})-f(\hat{\mathbf{x}})\| \leq\left(\max _{\mathbf{z} \in \mathcal{X}}\|\nabla f(\mathbf{z})\|\right)\|\mathbf{x}-\hat{\mathbf{x}}\|, \quad \forall \mathbf{x}, \hat{\mathbf{x}} \in \mathcal{X} .
$$

To solve this problem, we consider both deterministic and stochastic approaches. The deterministic approach utilize interval arithmetic for global optimization to find the best interval for $g(\mathbf{z}):=\max _{\mathbf{z} \in \mathcal{X}}\|\nabla f(\mathbf{z})\|$. The stochastic approach uses quasi random sampling with low discrepancy sequences [4] to approximate the global solution of $g(\mathbf{z})$. Other than Lipschitz nonlinearity, we plan to propose a unified framework to find such constants for other classes of nonlinear functions, including one-sided Lipschitz, quadratically inner-bounded, and bounded Jacobian.

\subsection{SCSP Strategies for NDN}

After the NDN's nonlinearity is identified, Lyapunov theory can then be exploited to construct SCSP formulations, given that most NDN can be expressed in the following form

$$
\dot{\mathbf{x}}(t)=\mathbf{A x}(t)+\mathbf{G} \mathbf{f}(\mathbf{x})+\mathbf{B} \Pi \mathbf{u}(t), \quad \mathbf{y}(t)=\Gamma \mathbf{C} \mathbf{x}(t),
$$

where the network is comprised of $N$ subsystems, each having its own states, control inputs, and outputs. The nonlinearity is represented by $\mathbf{f}(\mathbf{x})$ while matrices $\mathrm{A}, \mathrm{B}, \mathrm{C}, \mathrm{G}$ define the topology and parameters of the NDN. Matrices $\Pi$ and $\Gamma$ are diagonal with binary variables defining the selection of SC. As an illustration, let us revisit the previous example with $\beta$ as the Lipschitz constant of $\mathbf{f}$ where $\phi(\mathbf{x})=\mathbf{A x}(t)+\mathbf{G} \mathbf{f}(\mathbf{x})$. The observer design for this system with sensor selection problem can be posed as

$$
\begin{aligned}
\underset{\Gamma, \mathrm{P}, \mathrm{Y}, \kappa}{\operatorname{minimize}} & \mathbf{1}^{\top} \boldsymbol{\gamma} \\
\text { subject to } & {\left[\begin{array}{cc}
\mathbf{A}^{\top} \mathbf{P}+\mathrm{PA}-\mathrm{C}^{\top} \mathbf{\Gamma \mathrm { Y } ^ { \top }}-\mathrm{Y} \Gamma \mathrm{C}+\kappa \beta^{2} \mathbf{I} & \mathrm{PG} \\
\mathrm{G}^{\top} \mathbf{P} & -\kappa \mathrm{I}
\end{array}\right]<0 } \\
& \mathbf{P}>0, \kappa \geq 0, \gamma \in\{0,1\}^{N},
\end{aligned}
$$

where $\gamma=\operatorname{vec}(\Gamma)$ and in the case of our example, $N=4$. This problem can be cast as a MI-SDP by invoking the disjunctive programming principles and the Big-M method [6, 7], in which the resulting problem is similar to (3) by replacing $\mathrm{Y} \Gamma$ with a new variable $\mathrm{Q}$ and adding the constraints $\left|\mathrm{Y}_{i j}-\mathrm{Q}_{i j}\right| \leq M\left(1-\gamma_{j}\right)$ and $\left|Q_{i j}\right| \leq M \gamma_{j}$ for all $i, j$ with $M$ as a large positive constant. This problem can be solved with various methods, with $\mathrm{BnB}$ algorithm being the popular one. Although $\mathrm{BnB}$ algorithm theoretically is able to give optimal solutions, unfortunately, its computational time does not scale well with the number of variables, which in turn expose the limitation of this approach. With that in mind, we plan to explore different methods as well as develop faster computational algorithms which is tractable for large-scale CPS.

\section{REFERENCES}

[1] S. Contreras, P. Kachroo, and S. Agarwal. 2016. Observability and Sensor Placement Problem on Highway Segments: A Traffic Dynamics-Based Approach. IEEE Transactions on Intelligent Transportation Systems 17, 3 (March 2016), 848-858. https://doi.org/10.1109/TITS.2015.2491282

[2] A. Haber, F. Molnar, and A. E. Motter. 2018. State observation and sensor selection for nonlinear networks. IEEE Transactions on Control of Network Systems (2018), 1-1. https://doi.org/10.1109/TCNS.2017.2728201

[3] Sebastian A. Nugroho, Ahmad F. Taha, and Christian Claudel. 2018. A ControlTheoretic Approach for Modeling, Classification, and Robust Traffic Density Estimation. (2018). https://arxiv.org/pdf/1812.02128.pdf

[4] Sebastian A. Nugroho, Ahmad F. Taha, and Junjian Qi. 2019. Characterizing the Nonlinearity of Power System Generator Models. (2019). https://arxiv.org/pdf/ 1902.06025.pdf

[5] Junjian Qi, Kai Sun, and Wei Kang. 2015. Optimal PMU placement for power system dynamic state estimation by using empirical observability gramian. IEEE Transactions on power Systems 30, 4 (2015), 2041-2054.

[6] A. Taha, N. Gatsis, T. H. Summers, and S. A. Nugroho. 2018. Time-Varying Sensor and Actuator Selection for Uncertain Cyber-Physical Systems. IEEE Transactions on Control of Network Systems (2018), 1-1. https://doi.org/10.1109/TCNS.2018. 2873229

[7] J. A. Taylor, N. Luangsomboon, and D. Fooladivanda. 2017. Allocating Sensors and Actuators via Optimal Estimation and Control. IEEE Transactions on Control Systems Technology 25, 3 (May 2017), 1060-1067. https://doi.org/10.1109/TCST. 2016.2575799

[8] Stanislaw H. Zak. 2002. Systems and Control (1 ed.). Oxford University Press. 